\title{
Submitted: The usefulness of high-frequency ultrasonography Accepted: 20.11.2019 in the preoperative evaluation of vulvar cancer - a case series
}

Published: 31.12.2019

\section{Keywords}

high-frequency ultrasonography, vulvar cancer, vulva, ultrasonography

\author{
Michał Migda,2, Marian Stanisław Migda², Bartosz Migda³ \\ Marek Maleńczyk ${ }^{1}$
}

${ }^{1}$ Clinical Unit of Obstetrics, Women's Disease and Gynecological Oncology, United District Hospital, Collegium Medicum University of Nicolaus Copernicus in Torun, Torun, Poland

${ }^{2}$ Civis Vita Medical Center Torun, Poland

${ }^{3}$ Department of Diagnostic Imaging, Second Faculty of Medicine with the English Division and the Physiotherapy Division, Medical University of Warsaw, Warsaw, Poland

Correspondence: Michat Migda, Warszawska 20, 87-100 Torun; tel.: 566447607 , e-mail:mchmigda@gmail.com

DOI: $10.15557 / \mathrm{JoU} .2019 .0046$

\begin{abstract}
Vulvar cancer is a malignancy of female lower genital tract with incidence of 1:100,000 in Poland. The most common types of vulvar cancers are squamous cell carcinoma and basal cell carcinoma. This study was approved by the board of the Clinical Unit of Obstetrics, Women's Disease and Gynecological Oncology, United District Hospital, Collegium Medicum University of Nicolaus Copernicus in Torun, Poland. This is a tertiary reference center. We analyzed cases of postmenopausal women diagnosed with vulvar cancer referred to our clinic for surgery. The aim of the study was to compare $48 \mathrm{MHz}$ ultrasound images of vulvar cancer with histological images and assess the utility of high-frequency ultrasonography images in preoperative assessment. We examined 3 cases of squamous cell carcinoma and 1 case of basal cell carcinoma using $48 \mathrm{MHz}$ DermaView high-frequency mechanical probe. We described typical ultrasonographic features of vulvar carcinoma, such as echogenicity, depth of infiltration, as well as we discussed limitations of this transducer. The $48 \mathrm{MHz}$ frequency exhibits a promising value for the preoperative assessment of vulvar carcinoma. High-frequency ultrasonography can provide some important information, such as morphology, boundary, internal echo, and thus help choose adequate surgical approach.
\end{abstract}

\section{Introduction}

Vulvovaginal tissues are particularly susceptible to agerelated changes ${ }^{(1,2)}$. The histological, chemical, and immune changes that occur with aging increase the risk of vulvovaginal diseases in postmenopausal women ${ }^{(3)}$. At the cellular level, the highest estrogen-induced parakeratosis of vulvar stratum corneum is observed in the third decade of life, but is rare in the eighth decade ${ }^{(4)}$. Cell-mediated immunity is also diminished during menopause, which is caused by a $50 \%$ decrease of Langerhans cells in vulvar tissue, which increases the risk of infection and vulvar cancer ${ }^{(5)}$. Vulvar carcinogenesis is driven by chronic inflammatory conditions, autoimmune dysfunction, and human papillomavirus (HPV) disease ${ }^{(6)}$. Half of vulvar carcinomas are related to HPV infection suppressing Langerhans cell function ${ }^{(7)}$. More than $60 \%$ of vulvar SCCs develop after menopause due to impaired vulvovaginal immunity ${ }^{(8)}$. In Poland, vulvar cancer accounts for $1 \%$ of all malignancies. In 2016, there were only 498 cases reported in the National Cancer Registry ${ }^{(9)}$. Highfrequency ultrasonography (HFUS) is a technological 
advance, which is used in certain dermatological pathologies including skin cancer ${ }^{(10,11)}$. The diagnostic utility of HFUS, its noninvasiveness and reproducibility make it a useful tool in cancer diagnosis, mainly in melanoma and non-melanoma skin cancers (basal cell carcinoma, BCC), SCC, where strong correlations were found between histological and ultrasonographic values of tumor borders ${ }^{(12)}$. The aim of the study was to compare $48 \mathrm{MHz}$ ultrasound images of vulvar cancer with histological images.

\section{Materials and methods}

This study was approved by the board of Clinical Unit of Obstetrics, Women's Disease and Gynecological Oncology, United District Hospital, Collegium Medicum University of Nicolaus Copernicus in Torun, Poland. This is a tertiary reference center. We analyzed cases of postmenopausal women sent to our clinic for surgery due to diagnosed vulvar cancer. In this study, we performed high-frequency ultrasonography (HFUS) examinations using $48 \mathrm{MHz}$ DermaView high-frequency mechanical scanner (Dramiński S.A., Poland) prior to surgical excision. The collected specimens of altered tissue were formalin-fixed and paraffin-embedded. The paraffin-embedded blocks were cut into $4 \mu \mathrm{m}$ sections for a routine histological evaluation. We included 3 cases of SCC and 1 case of BCC in the study.

\section{Results}

\section{Squamous cell carcinoma (SCC)}

Cutaneous squamous cell carcinoma is an invasive malignant neoplasm of epidermal keratinocytes showing squamous phenotypic differentiation. SCC is the most common malignancy of the lower female genital tract and cervix. The peak incidence is after fifth decade of life in women, and approximately $60 \%$ of vulvar SCCs develop after menopause ${ }^{(13)}$. The classic presentation of genital SCC is a vulvar lump or mass. The lesion can be raised and may appear fleshy, ulcerated, leukoplakic, or warty (Fig. 1, Fig. 2, Fig. 3). We present 3 cases of SCC. All patients were over 70 years old. The main complain was pain and problems with urination (case 3 ) caused by urethral infiltration. First symptoms appeared approximately a year before in the form of a small red dot with itching. In gynecological examination, case 1 and 2 were solid tumors in the labia majora, with edema in the surrounding tissues. Palpation was difficult due to severe pain. Case 3 presented with massive edema of the vulva and a solid tumor infiltrating vagina and penetrating to the bone. In this case, metastatic tumors of $2-3 \mathrm{~cm}$ diameter were found bilaterally in the inguinal nodes. We performed ultrasound under anesthesia due to severe pain. According to literature, most SCCs are unifocal and develop on the labia majora ${ }^{(11)}$. In HFUS, SCC presents as a hypoechoic lesion with irregular borders. HFUS showed a clear picture of tumor margins

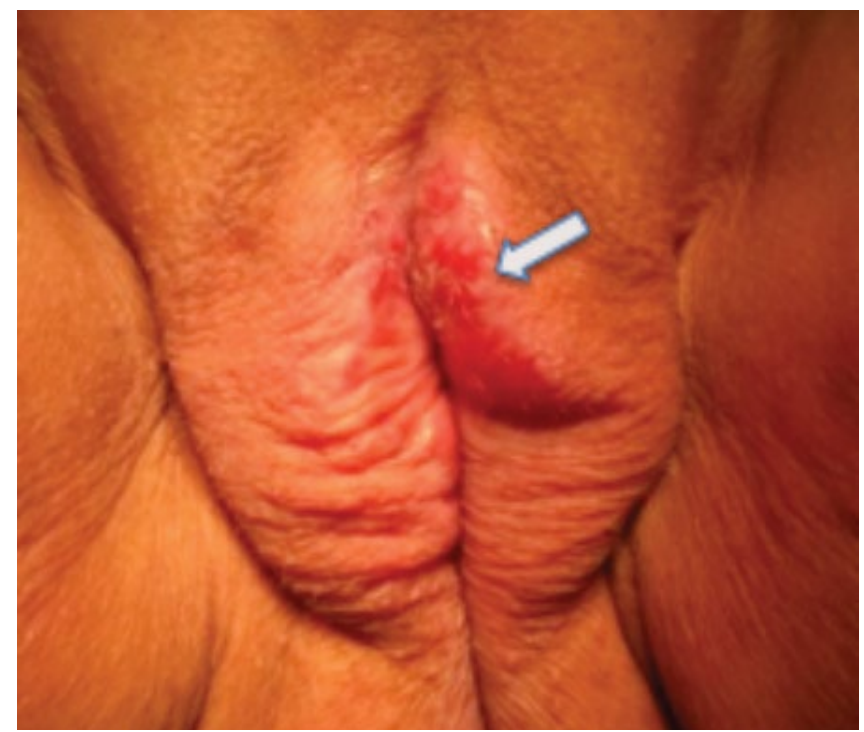

Fig. 1. SCC case 1 - a macroscopic image. Erytroplakia and skin edema on the labia majora (arrow)

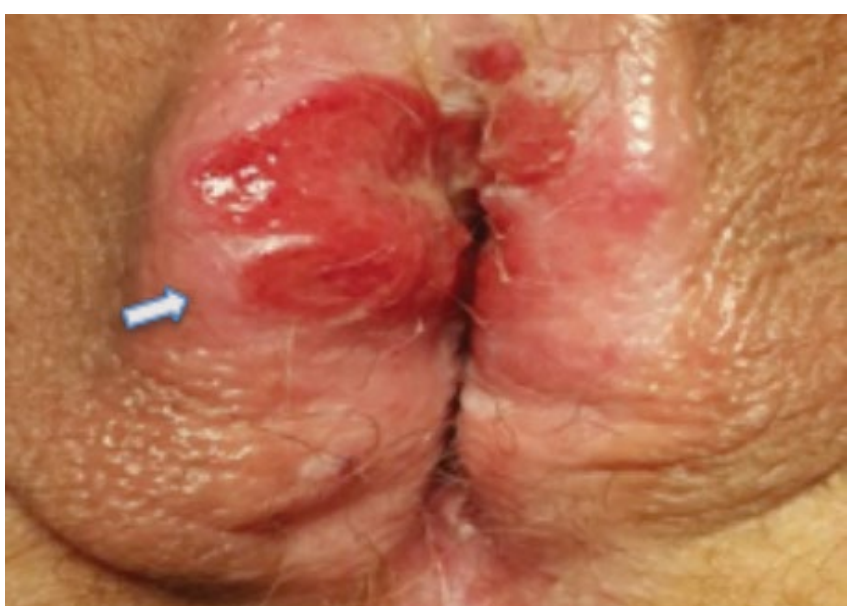

Fig. 2. SCC case 2 - a macroscopic image. Erytroplakia and skin edema on the labia majora (arrow)

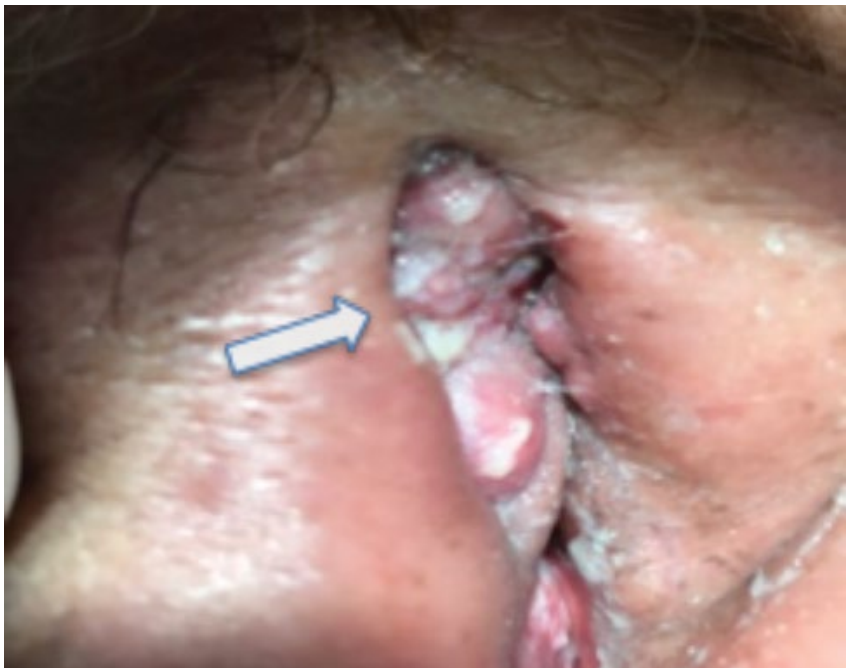

Fig. 3. SCC case 3- a macroscopic image. Solid mass arising from the labia majora (arrow) 


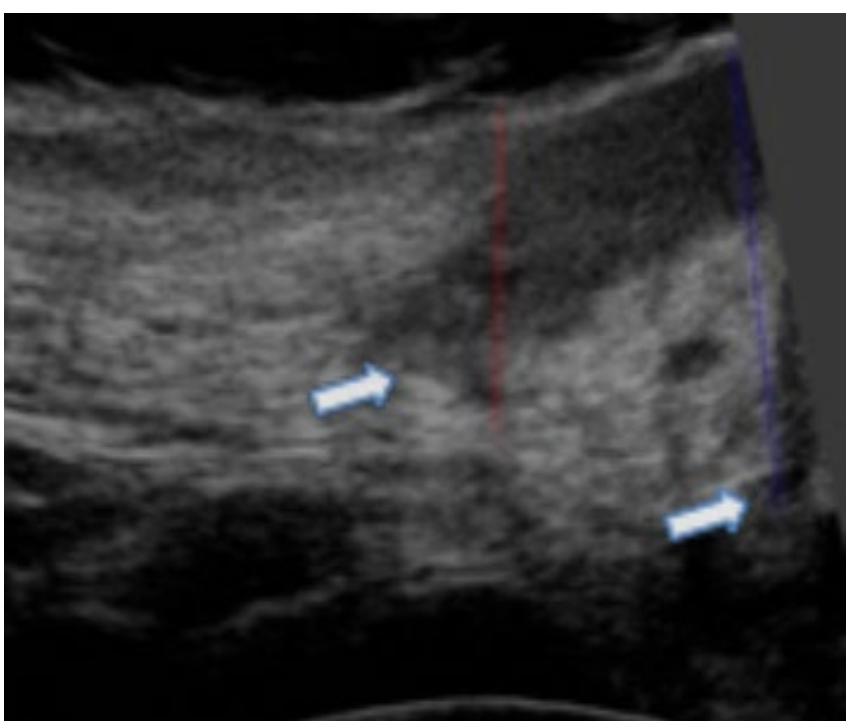

Fig. 4. HFUS image of SCC case 1. Hypoechoic irregular tumor borders invading the dermis. Tumor reaches subcutaneous tissue (arrows)

(Fig. 4, Fig. 5), and infiltration of the tumor penetrating much deeper into the skin, on contrary to case 2 where the margins were more blurry. Since thickness and depth of invasion are important predictors of metastases, the lesion should be followed along entire course. (Fig. 5, Fig 6). Typical histological images present atypical squamous cells, multiple mitotic figures and pseudoepitheliomatous hyperplasia. (Fig. 7, Fig. 8)

\section{Basal cell carcinoma (BCC)}

BCC is the most common malignant tumor of the skin (Fig. 9). BCC is characterized by an extremely rare metastasis and the capacity for extensive growth ${ }^{(14)}$. There are several clinical forms of BCC: superficial, nodular, ulcerative, sclerodermiform and their combined variants ${ }^{(15)}$. The method of treatment involves complete excision with most acceptable cosmetic result ${ }^{(15)}$. HFUS provides high resolution allowing to

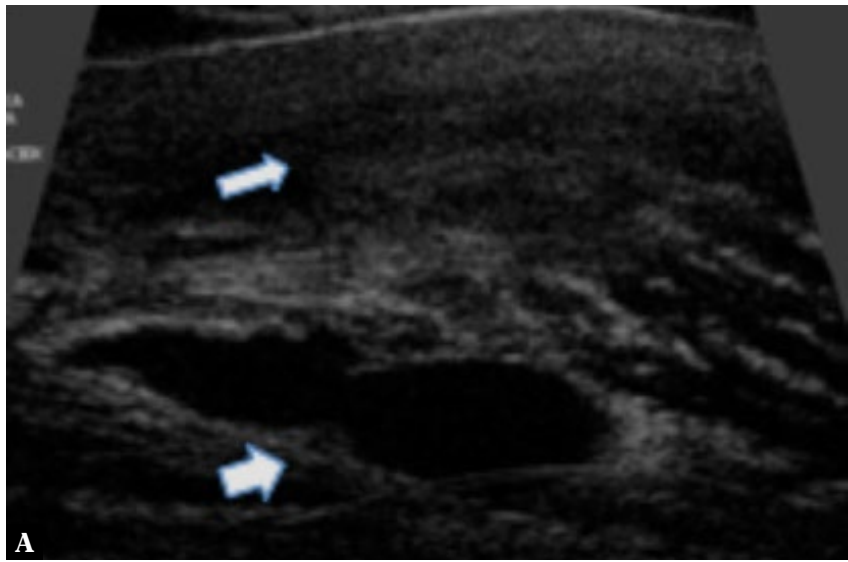

differentiate between the epidermis, dermis and subcutaneous fatty tissue. At ultrasound scanning, a superficial BCC appears as a hypoechoic zone located beneath the epidermis, with an elongated shape and distinct contours (Fig. 10). Corresponding histological images show a tumor arising form the epidermis, composed of basaloid cells (Fig. 11).

\section{Discussion}

Vulvar cancer accounts for over 5\% of gynecological malignancies and approximately $1 \%$ of malignancies in women. SCC is the most common vulvar malignancy (over $90 \%$ of malignant tumors $)^{(16)}$. Literature reports on the use of noninvasive techniques in the differential diagnosis of intraepithelial neoplasia and SCC are sparse and based mainly on case reports ${ }^{(17)}$. Dermatoscopy has shown some diagnostic utility in Bowen's disease of the vulva that progressed to invasive $\mathrm{SCC}^{(18)}$. Optical coherence tomography is another diagnostic tool to differentiate between epidermal thickness of normal vulvar tissue and epidermal thickness of vulvar intraepithelial neoplasia ${ }^{(19)}$. Literature data show that optical coherent tomography may be applied for determining appropriate surgical margins in patients with vulvar SCC ${ }^{(20)}$. The value of high-frequency ultrasonography in clinical dermatology is still being debated. All nonmelanoma skin cancer lesions appeared hypoechoic in HFUS, suggesting that this method alone is not suitable for differential diagnosis ${ }^{(17)}$. This is similar to our observations. In our study, SCC tumors were hypoechoic. In all SCC HFUS images, the margins were irregular and in one case (case 2) segmentally difficult to determine. However, in case 1 and 2 we could assess the margins what helped us to plan excisions, basing on the acquired images. Case 3 was an advanced infiltrating SCC, and evaluation of tumor margins was not possible. Nevertheless, in case 1 and 2, the depth of invasion and infiltration of the subcutaneous tissue was visualized. Macroscopic assessment and palpation underestimated the tumor infiltration. We increased the margin of excision based on the ultrasonographic image. Due to the size of the tumor it is impossible to visualize the whole structure. The assessment of tumor margins in

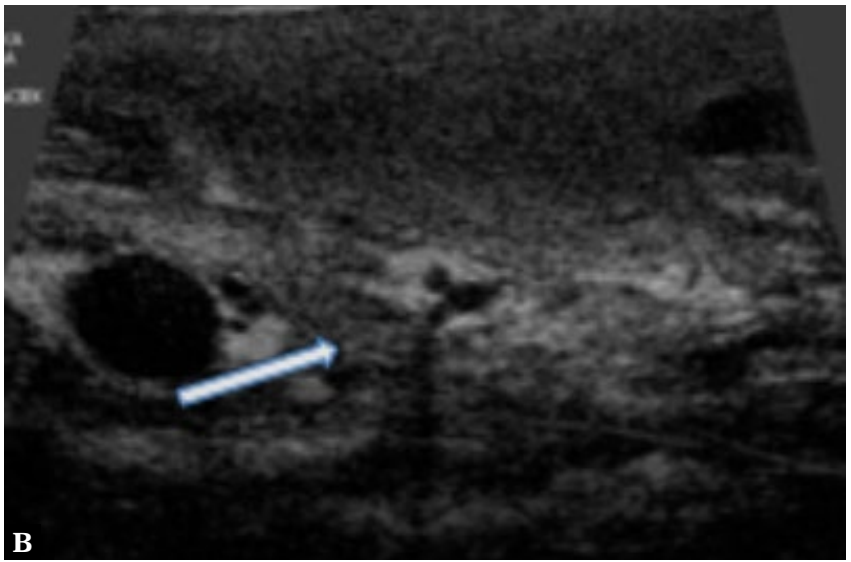

Fig. 5. HFUS SCC case 3. A. Hipoechoic, non-homogeneous structure of the tumor (short arrow). An enlarged blood vessel with edema (short thick arrow). B. Deep tumor invasion. Tumor margins are fused with unchanged surrounded soft tissues (long arrow) 


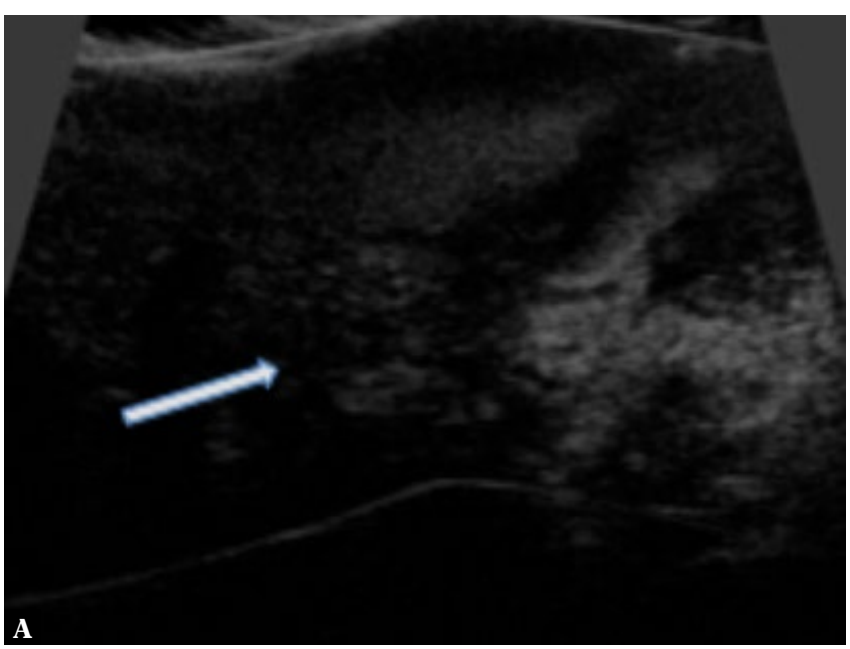

Fig. 6. HFUS image of SCC case 2. A. Very dense and hypoechoic B. Irregular, partially blurred tumor margins (short arrow)

cases 1 and 2 was possible, but time-consuming. The location is also challenging due to irregular shape of the vulva and pain during examination. A complete ultrasonographic examination was possible during anesthesia in all cases.

$\mathrm{BCC}$ is the most common cutaneous malignant tumor. According to recent literature, most studies regarding HFUS imaging of BCC are based on locations other than vulva. There are several clinical forms of BCC: superficial, nodular, ulcerative, sclerodermiform and their combined variants ${ }^{(15)}$. In earlier studies, the use of a $20 \mathrm{MHz}$ frequency probe allowed to detect BCC as a hypoechoic zone with local thickening of the epidermis and the dermis ${ }^{(21)}$. All clinical variants of BCCs were defined as a hypoechoic zone located directly beneath the epidermis and propagating into the dermis at a varying depth, mostly round or oval ${ }^{(22)}$. Similar HFUS images were obtained in our study using a $48 \mathrm{MHz}$ probe.

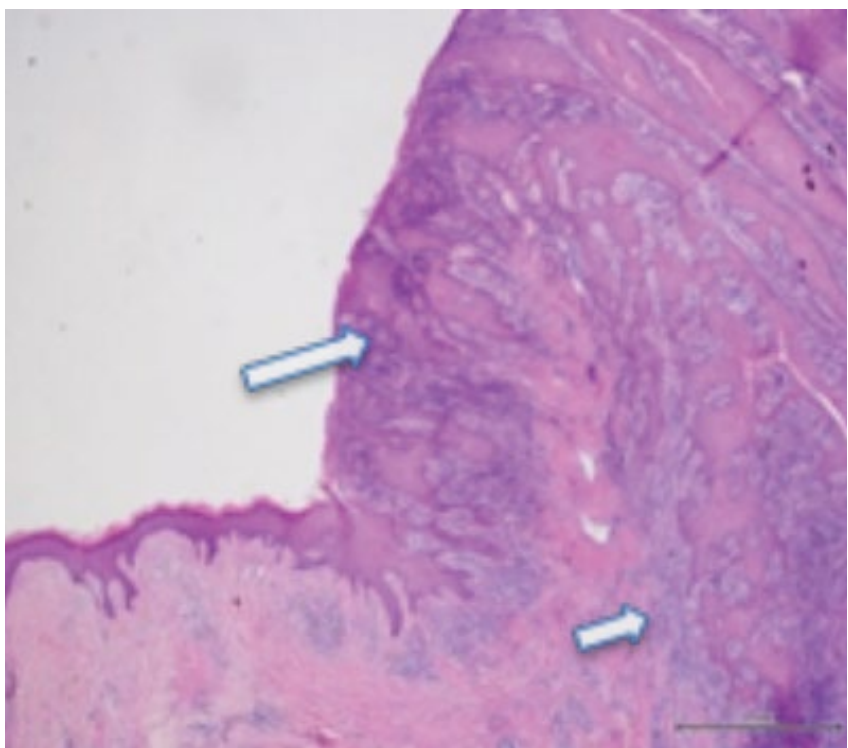

Fig. 7. SCC case 1- a microscopic image of SCC. Histopathology shows pseudoepitheliomatous hyperplasia (long arrow), atypical squamous cells, and multiple mitotic figures (short arrow) (H and E)

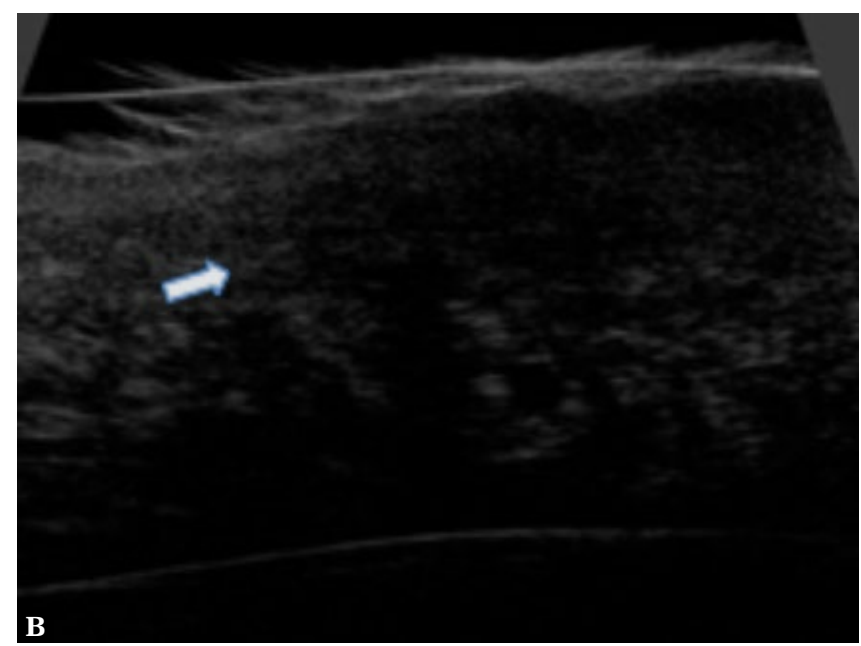

structure of the tumor, reaching subcutaneous tissue (long arrow).

The solid structure of the tumor is the main challenge during examination. It is possible to assess tumor margins and depth of invasion precisely. Due to the histological structure of the tumor and the probe frequency used in this study (48 $\mathrm{MHz}$ ), it was unable to get more precise information about tumor echostucture, which was visible as a hypoechoic lesion. Other authors reported using $22 \mathrm{MHz}$ transducers and described BCC lesions as heterogeneous, hypoechoic lesions in the dermis with regular contour and large, hyperechoic internal signals ${ }^{(23)}$. Wang at al. suggest that hyperechoic spots are an important ultrasonographic feature of BCC lesions. In his study, $76 \%$ of BCC cases (31/46) displayed hyperechoic spots ${ }^{(24)}$. In our study, we did not visualize these features using a $48 \mathrm{MHz}$ transducer. On the other hand, HFUS could precisely show the deep structure of the lesion, and provide important information for further decisions on, for example, increasing the margin of excision.

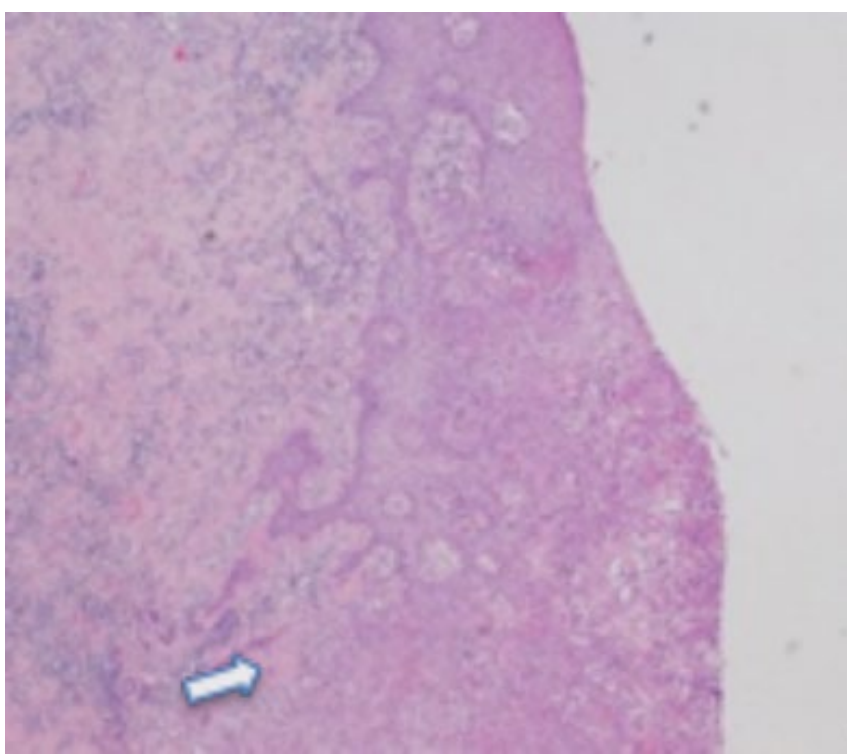

Fig. 8. SCC case 2. Microscopic image of SCC, atypical squamous cells, and multiple mitotic figures (arrow) $(H$ and $E)$ 


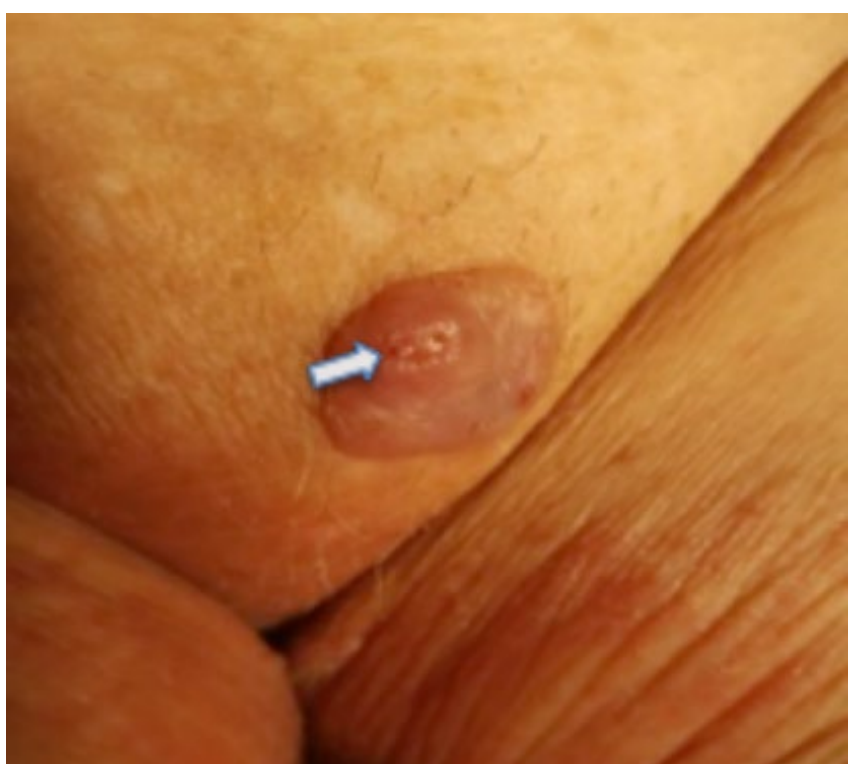

Fig. 9. BCC-macroscopic image. Nodular type on the mons pubis with a small erosion on the top (arrow)

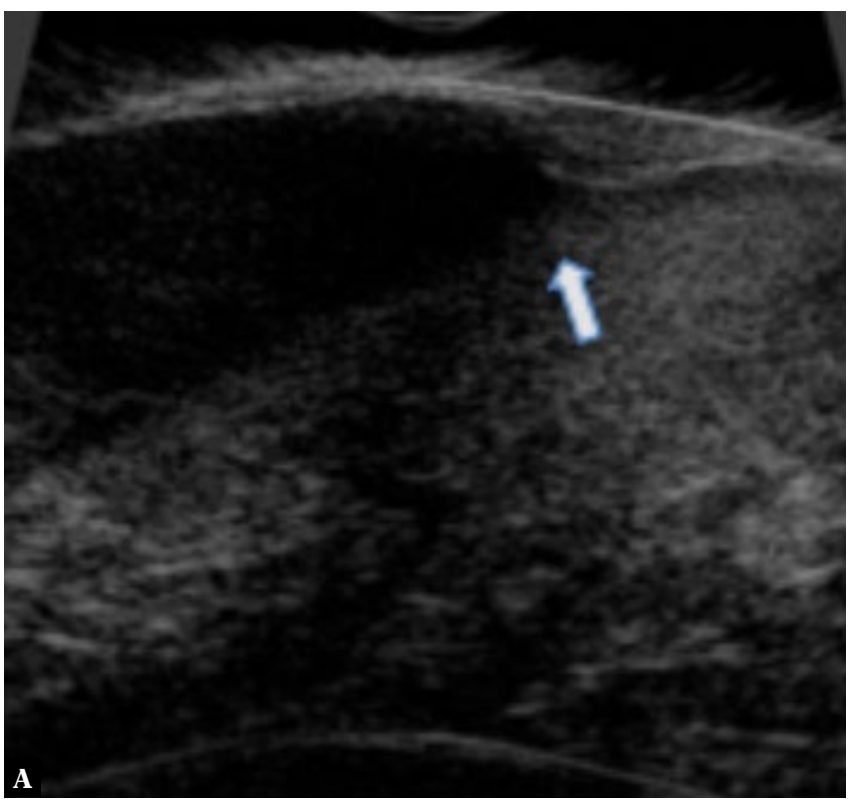

Fig. 10. A. A HFUS BCC image. Hypoechoic structure, sharp margins (arrow). B. Lower margin well defined (long arrow)

Our study had some limitations. Firstly, we used a small number of cases due to the rare occurrence of this malignancy. Secondly, all cases were referred to our center at an advanced stage of the disease. Thirdly, HFUS is a mechanical probe, allowing only a gray scale assessment. In our opinion, additional options like Doppler ultrasound, microflow imaging, elastography would bring useful information about vulvar cancer.

\section{Conclusion}

HFUS seems a promising tool for the preoperative assessment of BCC and SCC. High-frequency probes can provide

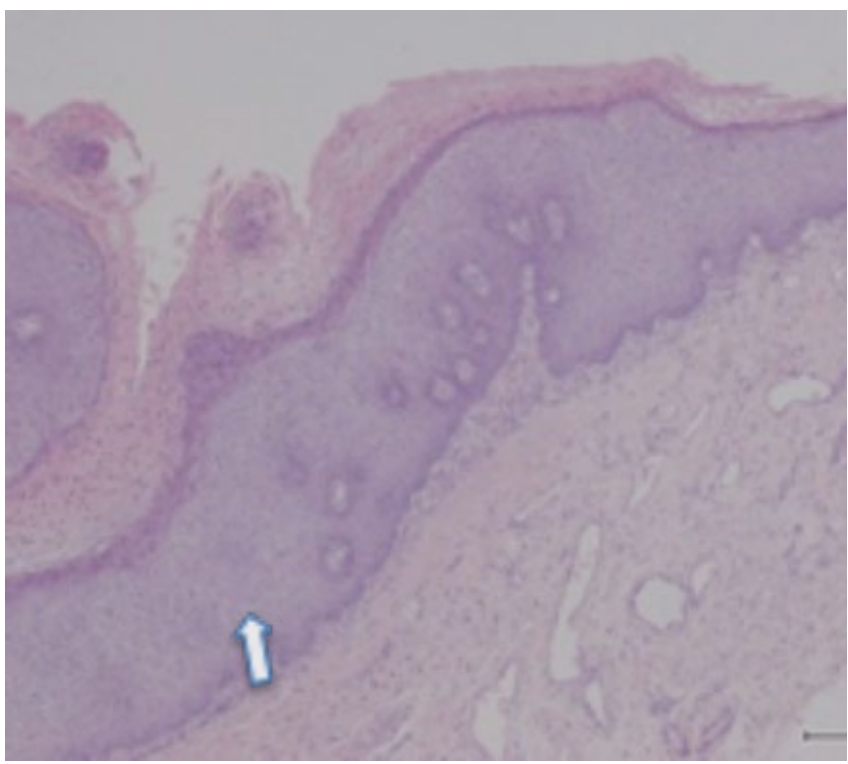

Fig. 11. A microscopic image of BCC. Tumor arising from the epidermis, composed of basaloid cells (arrow)

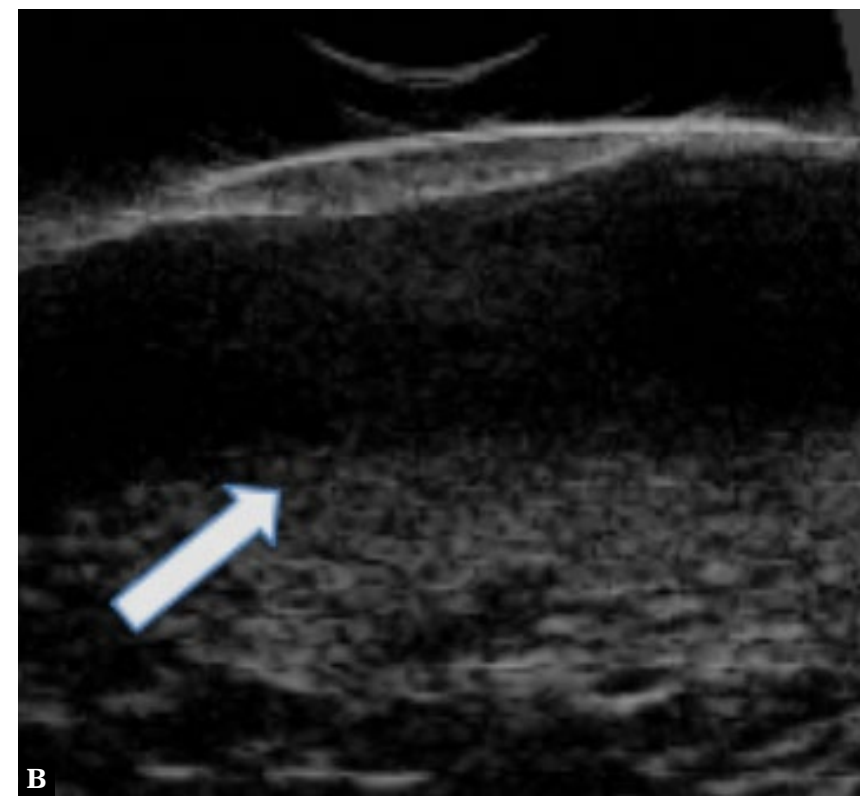

some important information, such as morphology, margins, and internal echo, contributing to the choice of an adequate surgical approach. The rapid progress in non-invasive skin imaging indicates that preoperative evaluation will be soon incorporated in the daily practice.

\section{Conflict of interest}

Authors do not report any financial or personal connections with other persons or organizations, which might negatively affect the contents of this publication and/or claim authorship rights to this publication. 


\section{References}

1. Kamarashev JA, Vassileva SG: Dermatologic diseases of the vulva. Clin Dermatol 1997; 15: 53-65.

2. Matthews N, Wong V, Brooks J, Kroumpouzos G: Genital diseases in the mature woman. Clin Dermatol 2018; 36: 208-221.

3. Stiles M, Redmer J, Paddock E, Schrager S: Gynecologic issues in geriatric women. J Womens Health (Larchmt) 2012; 21: 4-9.

4. Harper WF, McNicol EM: A histological study of normal vulval skin from infancy to old age. Br J Dermatol 1977; 96: 249-253.

5. Edwards JN, Morris HB: Langerhans' cells and lymphocyte subsets in the female genital tract. Br J Obstet Gynaecol 1985; 92: 974-982.

6. Chokoeva AA, Tchernev G, Castelli E, Orlando E, Verma SB, Grebe M et al.: Vulvar cancer: a review for dermatologists. Wien Med Wochenschr 2015; 165: 164-177.

7. Preti M, Scurry J, Marchitelli CE, Micheletti L: Vulvar intraepithelial neoplasia. Best Pract Res Clin Obstet Gynaecol 2014; 28: 1051-1062.

8. Akhtar-Danesh N, Elit L, Lytwyn A: Trends in incidence and survival of women with invasive vulvar cancer in the United States and Canada: a population-based study. Gynecol Oncol 2014; 134: 314-318.

9. Wojciechowska U, Czaderny K, Ciuba A, Olasek P, Didkowska J: Cancer in Poland in 2016. Biuletyn. Warszawa 2018.

10. Migda MS, Migda M, Migda B, Słapa RZ, Mlosek RK: Feasibility of using high-frequency skin ultrasound (HFSU) in vulvar skin assessment - initial report with the description of HFSU anatomy. Ginekol Pol 2016; 87: 19-25.

11. Bard RL: High-frequency ultrasound examination in the diagnosis of skin cancer. Dermatol Clin 2017; 35: 505-511.

12. Polańska A, Bowszyc-Dmochowska M, Olek-Hrab K, Adamski Z, Żaba R, Dańczak-Pazdrowska A: High-frequency ultrasonography a new quantitative method in evaluation of skin lymphomas-First comparative study in relation to histopathology. Skin Res Technol 2019; 25: 720-724.

13. Vitale SG, Valenti G, Biondi A, Rossetti D, Frigerio L: Recent trends in surgical and reconstructive management of vulvar cancer: review of literature. Updates Surg 2015; 67: 367-371.
14. Nakayama M, Tabuchi K, Nakamura Y, Hara A: Basal cell carcinoma of the head and neck. J Skin Cancer 2011; 2011: 496910.

15. Marzuka AG, Book SE: Basal cell carcinoma: pathogenesis, epidemiology, clinical features, diagnosis, histopathology, and management. Yale J Biol Med 2015; 88: 167-179.

16. Alkatout I, Schubert M, Garbrecht N, Weigel MT, Jonat W, Mundhenke C et al:: Vulvar cancer: epidemiology, clinical presentation, and management options. International journal of women's health 2015; 7: 305313.

17. Warszawik-Hendzel O, Olszewska M, Maj M, Rakowska A, Czuwara J, Rudnicka L: Non-invasive diagnostic techniques in the diagnosis of squamous cell carcinoma. J Dermatol Case Rep 2015; 9: 89-97.

18. Lazaridou E, Fotiadou C, Giannopoulou C, Ioannides D: Persistent erythematous lesion of the vulva: a diagnostic and treatment challenge. $\mathrm{J}$ Drugs Dermatol 2012; 11: 110-112.

19. Wessels R, de Bruin DM, Faber DJ, van Boven HH, Vincent AD, van Leeuwen TG et al.: Optical coherence tomography in vulvar intraepithelial neoplasia. J Biomed Opt 2012; 17: 116022.

20. Wessels R, van Beurden M, de Bruin DM, Faber DJ, Vincent AD, Sanders $\mathrm{J}$ et al.: The value of optical coherence tomography in determining surgical margins in squamous cell carcinoma of the vulva: a singlecenter prospective study. Int J Gynecol Cancer 2015; 25: 112-118.

21. Bezugly A: High frequency ultrasound study of skin tumors in dermatological and aesthetic practice. Med Ultrason 2015; 17: 541-544.

22. Khlebnikova AN, Molochkov VA, Selezneva EV, Belova LA, Bezugly A, Molochkov AV: Ultrasonographic features of superficial and nodular basal cell carcinoma. Med Ultrason 2018; 20: 475-479.

23. Barcaui Ede O, Carvalho AC, Valiante PM, Barcaui CB: High-frequency ultrasound associated with dermoscopy in pre-operative evaluation of basal cell carcinoma. An Bras Dermatol 2014; 89: 828-831.

24. Wang SQ, Liu J, Zhu QL, Zhao CY, Qu T, Li F et al.: High-frequency ultrasound features of basal cell carcinoma and its association with histological recurrence risk. Chin Med J (Engl) 2019; 132: 2021-2026. 